\title{
Can extent of high grade dysplasia in Barrett's oesophagus predict the presence of adenocarcinoma at oesophagectomy?
}

\author{
M S Dar, J R Goldblum, T W Rice, G W Falk
}

Gut 2003;52:486-489

See end of article for authors' affiliations

Correspondence to: Dr G W Falk, Department of Gastroenterology and Hepatology, Desk A- 30, Cleveland Clinic

Foundation, 9500 Euclid Avenue, Cleveland, Ohio 44195, USA;

falkg@ccf.org

Accepted for publication 6 November 2002
Background: Optimal management of Barrett's oesophagus complicated by high grade dysplasia is controversial. Recently, the extent of high grade dysplasia was described as a predictor of subsequent development of cancer in patients undergoing continued surveillance. However, there is no universal agreement on the definition of extent of high grade dysplasia.

Aim: To determine if extent of high grade dysplasia in Barrett's oesophagus is a predictor of the presence of adenocarcinoma at the time of oesophagectomy.

Methods: Forty two patients with Barrett's oesophagus and high grade dysplasia who underwent oesophagectomy between 1985 and 1999 were identified from a prospective database. All pathological specimens, including preoperative endoscopic biopsies and post-oesophagectomy sections, were reviewed in a blinded fashion by one expert gastrointestinal pathologist to determine the extent of high grade dysplasia. The extent of high grade dysplasia was defined using two different criteria, one from the Cleveland Clinic and one from the Mayo Clinic.

Results: Twenty four of 42 patients (57\%) had unsuspected cancer at the time of oesophagectomy. Using the Cleveland Clinic definition, 10 of 21 (48\%) patients with focal high grade dysplasia had carcinoma compared with 14 of 21 patients (67\%) with diffuse high grade dysplasia $(p=0.35)$. Using the Mayo Clinic definition, adenocarcinoma was found in five of seven (72\%) patients with focal high grade dysplasia compared with 19 of 35 (54\%) with diffuse high grade dysplasia $(p=0.68)$.

Conclusions: The extent of high grade dysplasia, regardless of how it is defined, does not predict the presence of unsuspected adenocarcinoma at oesophagectomy. There is no evidence as yet that the extent of high grade dysplasia can be used as a basis for decision making in these patients.
$\mathrm{H}$ gh grade dysplasia in Barrett's oesophagus is associated with an increased risk for invasive oesophageal adenocarcinoma. ${ }^{1-15}$ However, the optimal management of these patients remains controversial. Some advocate immediate surgery in those with high grade dysplasia because of the possibility of unsuspected cancer at the time of oesophagectomy..$^{2-7}$ 9-11 Others recommend continued endoscopic surveillance, reserving oesophagectomy for patients with clearly demonstrated cancer. ${ }^{14} 16$ Still others advocate endoscopic ablation therapy. ${ }^{17} 18$

Resolution of this dilemma is of more than academic interest. Oesophageal adenocarcinoma is an often lethal disease and survival is stage dependent. Lymph node metastases are encountered in up to $6 \%$ of patients with intramucosal carcinoma and $24 \%$ of patients with submucosal carcinoma. ${ }^{19-21}$ Patients with high grade dysplasia are often elderly with comorbid conditions. Even in the best of surgical hands, oesophagectomy for superficial adenocarcinoma is associated with a mortality of at least $2 \% .{ }^{19}$ Thus markers of increased cancer risk in patients with high grade dysplasia would be welcome in an effort to eliminate prophylactic oesophagectomies. To date, there are no clear factors that predict which patients with high grade dysplasia will have adenocarcinoma at the time of oesophagectomy.

Recently, the extent of the Barrett's segment involved with high grade dysplasia was identified as a potential risk factor for the subsequent development of adenocarcinoma. ${ }^{13}$ This concept raises several questions. Is there a threshold burden of high grade dysplasia above which concern for invasive cancer would be raised and below which concern would be lowered? How does one quantify the extent of high grade dysplasia? There are currently no uniform criteria for defining the extent of high grade dysplasia. The definition of extent of high grade dysplasia used by Buttar et al differs from what we and others use by focusing on the number of crypts involved, as well as the number of biopsy specimens with high grade dysplasia. ${ }^{13}$ Thus the aim of this study was to determine if the extent of high grade dysplasia, using two different criteria, is associated with a different risk for cancer at the time of oesophagectomy.

\section{METHODS \\ Patients}

This was a cohort study of all patients undergoing oesophagectomy for high grade dysplasia at the Cleveland Clinic Foundation between 1985 and 1999. Patients were identified from an Institutional Review Board approved prospective surgical database of all patients undergoing oesophageal surgery. Patients excluded were those with an obvious mass at the time of endoscopy and those with a preoperative diagnosis of intramucosal or submucosal adenocarcinoma.

Medical records at the time of surgery were reviewed for demographic information. Endoscopic findings were noted prior to surgery. These included length of Barrett's epithelium, hiatal hernia size, and presence of mucosal abnormalities.

\section{Endoscopy}

A biopsy protocol using a standard biopsy forceps was used between 1986 and 1992. In 1993, a modified "Seattle" biopsy protocol was adopted and remained in effect through 1999, as outlined elsewhere. ${ }^{5}{ }^{10}$ The standard biopsy protocol obtained biopsy specimens using a standard biopsy forceps at $2 \mathrm{~cm}$ intervals along the entire length of the Barrett's epithelium, 
beginning at the end of the tubular oesophagus just above the proximal margin of the gastric folds, and continuing to the new squamocolumnar junction. The modified "Seattle" protocol used large capacity spiked biopsy forceps (Olympus FB-13K or 50U) to obtain biopsies from four quadrants at intervals of $2 \mathrm{~cm}$ or less along the entire length of Barrett's epithelium, beginning at the end of the tubular oesophagus just above the proximal margin of the gastric folds, and continuing to the new squamocolumnar junction. In both groups, biopsy specimens from each level were placed in separate containers to better document the exact level of an abnormality. Additional biopsy specimens were obtained from any ulcer, erosion, plaque, nodule, stricture, or other luminal irregularity, and submitted separately.

\section{Pathology}

Biopsy and oesophageal resection specimens were prepared as described elsewhere. ${ }^{10}$ Biopsy specimens were fixed in Hollande's solution and a minimum of three step sections were stained: two with haematoxylin and eosin, and one with Alcian blue/periodic acid Schiff combination stain.

Surgical specimens fixed in Hollande's solution were extensively sampled. In each case, all resected lymph nodes were examined. Oesophageal resection specimens were evaluated using a standardised protocol for resection margins, the oesophageal body, the gastro- oesophageal junction, and regional lymph nodes. When gross lesions were identified in the oesophagectomy specimens, up to five sections of each lesion, including the area of deepest penetration of the oesophageal wall, were evaluated. All separately resected lymph nodes and all lymph nodes grossly identified in the resection specimen were evaluated. When small enough, the entire lymph node was submitted and two levels examined histologically. Larger lymph nodes were bisected and two levels from each section examined. In the absence of a grossly identifiable lesion, the entire Barrett's oesophagus region was submitted for histological evaluation.

High grade dysplasia in Barrett's oesophagus was defined by an established classification scheme. ${ }^{20}$ High grade dysplasia is characterised by marked architectural and/or cytological abnormalities, either of which could predominate. Architectural abnormalities include budded, branched, crowded, or irregularly shaped glands, papillary extensions into gland lumina, and a mucosal villiform configuration. Nuclear features include marked variation in size and shape, nuclear or nucleolar enlargement, increased nuclear to cytoplasmic ratio, hyperchromatism, and abnormal mitoses. Nuclear alterations are always present on the surface epithelium.

The extent of high grade dysplasia was determined using two separate sets of criteria: one from the Cleveland Clinic and one from the Mayo Clinic. The Cleveland Clinic definition of focal high grade dysplasia was the presence of high grade dysplasia at only one level of the oesophagus - that is, high grade dysplasia present in biopsy specimens obtained at $38 \mathrm{~cm}$ but not at $36 \mathrm{~cm}$ or $34 \mathrm{~cm}$ in the Barrett's segment. Diffuse high grade dysplasia was defined as the presence of high grade dysplasia at multiple levels of the oesophagus-that is, in more than one specimen taken from the patient.

The Mayo Clinic definition of focal high grade dysplasia was the presence of high grade dysplasia limited to five or fewer crypts and associated tubuloalveolar acini in one biopsy specimen of the entire set of surveillance biopsies. ${ }^{13}$ Diffuse high grade dysplasia was defined as high grade dysplasia involving more than five crypts and associated tubuloalveolar acini in one endoscopic biopsy specimen or involvement of more than one biopsy specimen with any amount of high grade dysplasia. ${ }^{13}$

Intramucosal carcinoma was defined as carcinoma cells extending beyond the basement membrane into the lamina propria or muscularis mucosae, but not beyond. Submucosal
Table 1 Comparison of focal versus diffuse high grade dysplasia populations: Cleveland Clinic criteria

\begin{tabular}{|c|c|c|c|}
\hline & $\begin{array}{l}\text { Focal high } \\
\text { grade } \\
\text { dysplasia } \\
\text { (n=21) }\end{array}$ & $\begin{array}{l}\text { Diffuse high } \\
\text { grade } \\
\text { dysplasia } \\
\text { (n=21) }\end{array}$ & $p$ Value \\
\hline $\operatorname{Sex}(M / F)$ & $17 / 4$ & $20 / 1$ & 0.34 \\
\hline Age $(y)$ & $60.2(11.5)$ & $58.7(9.8)$ & 0.64 \\
\hline Barrett's length $(\mathrm{cm})$ & $7.2(3.7)$ & $9.3(3.4)$ & 0.06 \\
\hline Hiatal hernia length $(\mathrm{cm})$ & $3.9(1.2)$ & $3.8(1.9)$ & 0.43 \\
\hline Unsuspected cancer & 10 & 14 & 0.350 \\
\hline $\begin{array}{l}\text { Intramucosal/submucosal } \\
\text { cancer }\end{array}$ & $9 / 1$ & $11 / 3$ & 0.61 \\
\hline Nodularity & 5 & 5 & 0.83 \\
\hline
\end{tabular}

carcinoma was defined as infiltration of carcinoma cells beyond the muscularis mucosae into the submucosa with an associated desmoplastic response.

All specimens from all patients, including preoperative endoscopic biopsy and postoperative oesophagectomy specimens, were re-reviewed in a blinded fashion by one expert gastrointestinal pathologist (JRG). Any patient with a preoperative biopsy showing intramucosal or submucosal carcinoma was excluded.

\section{Surgery}

All patients underwent oesophagectomy with regional lymphadenectomy or lymph node sampling and gastric replacement. Oesophagogastric anastomoses were accomplished in the neck. Thirty four patients underwent transhiatal oesophagectomy whereas eight underwent a transthoracic oesophagectomy.

\section{Statistical analysis}

Continuous variables were analysed using the two sample $t$ test whereas categorical data were compared using Fisher's exact or $\chi^{2}$ test. A p value $<0.05$ was considered to be significant.

\section{RESULTS}

Demographics of the study population are shown in table 1. A total of 42 patients underwent surgery for high grade dysplasia. Unsuspected cancer was detected in 24 of 42 patients $(57 \%)$ at the time of oesophagectomy. Of these 24 cancers, 20 were intramucosal and four were submucosal. Lymph node metastases were detected in none of the patients with intramucosal adenocarcinoma and in one patient with submucosal adenocarcinoma.

Using the Cleveland Clinic criteria, adenocarcinoma was present in the oesophagectomy specimen in 10 of 21 patients $(48 \%)$ with focal high grade dysplasia compared with 14 of 21 $(67 \%)$ patients with diffuse high grade dysplasia $(p=0.35)$ (table 1). There were no differences in sex, age, length of Barrett's oesophagus, hiatal hernia size, or nodularity between these two groups.

Using the Mayo Clinic criteria, there was a decrease in the number of patients classified as having focal high grade dysplasia to seven. However, adenocarcinoma was found in the oesophagectomy specimen in five of seven $(72 \%)$ patients with focal high grade dysplasia compared with 19 of 35 (54\%) patients with diffuse high grade dysplasia $(\mathrm{p}=0.68)$ (table 2$)$. There were no differences in age, length of Barrett's epithelium, hiatal hernia size, or nodularity between these two groups.

\section{DISCUSSION}

The optimal management of high grade dysplasia in Barrett's oesophagus remains a clinical dilemma. Factors contributing 
Table 2 Comparison of focal versus diffuse high grade dysplasia populations: Mayo Clinic criteria

\begin{tabular}{llll}
\hline & $\begin{array}{l}\text { Focal high } \\
\text { grade dysplasia } \\
(\mathrm{n}=7)\end{array}$ & $\begin{array}{l}\text { Diffuse high } \\
\text { grade dysplasia } \\
(\mathrm{n}=35)\end{array}$ & $\mathrm{p}$ Value \\
\hline Sex (M/F) & $6 / 1$ & $31 / 4$ & 1.00 \\
Age (y) & $56.9(6.5)$ & $59.9(11.2)$ & 0.49 \\
Barrett's length (cm) & $8.9(2.8)$ & $8.2(3.8)$ & 0.66 \\
Hiatal hernia length (cm) & $4.0(1.0)$ & $3.8(1.7)$ & 0.78 \\
$\begin{array}{l}\text { Unsuspected cancer } \\
\text { Intramucosal/submucosal }\end{array}$ & $5 / 0$ & 19 & 0.68 \\
$\quad$ cancer & 5 & $15 / 4$ & 0.54 \\
Nodularity & 2 & 8 & 1.00 \\
\hline Results are expressed as mean (2 SD). & & \\
\hline
\end{tabular}

to this include: (a) difficulty in detecting unsuspected cancer with current biopsy protocols, (b) interobserver variability among pathologists in the recognition of high grade dysplasia, and (c) the uncertain natural history of high grade dysplasia.

Unsuspected carcinoma is found at the time of oesophagectomy in approximately $40 \%$ of patients with a preoperative diagnosis of high grade dysplasia. ${ }^{5022}$ Our group has previously reported that $36 \%$ of high grade dysplasia patients with no obvious mass have unsuspected carcinoma at the time of oesophagectomy. ${ }^{10}$ In that study, no differences were apparent in the finding of unsuspected carcinoma regardless of whether a conventional or a large capacity "jumbo" biopsy forceps was used preoperatively. In contrast, others have advocated a rigorous biopsy protocol employing four quadrant biopsies at $1 \mathrm{~cm}$ intervals using jumbo biopsy forceps that reliably detects carcinoma, and thereby obviates the need for prophylactic oesophagectomy. ${ }^{16}{ }^{23}$ The University of Washington group recently described their experience in 45 Barrett's oesophagus patients with high grade dysplasia in whom cancer was eventually detected with this aggressive endoscopic biopsy protocol. ${ }^{23}$ Four quadrant biopsies at $2 \mathrm{~cm}$ intervals with the same biopsy forceps would have missed $50 \%$ of cancers in these patients. ${ }^{23}$ Eighty two per cent of cancers were found at a single $1 \mathrm{~cm}$ level of the oesophagus and $69 \%$ were found only in a single biopsy specimen.

While a meticulous biopsy protocol may detect unsuspected cancer preoperatively, problems continue in the pathological interpretation of high grade dysplasia, as well as the ability to differentiate between high grade dysplasia and intramucosal carcinoma. In a recent study by Montgomery et al, interobserver agreement for interpretation of high grade dysplasia by experienced gastrointestinal pathologists yielded a kappa score of only 0.43 , a moderate agreement. ${ }^{24}$ Is pathological interpretation improved by examination of oesophagectomy specimens? In a study of oesophagectomy specimens from patients with Barrett's oesophagus and high grade dysplasia or superficial carcinoma, the interobserver agreement for distinguishing high grade dysplasia from intramucosal carcinoma between two experienced gastrointestinal pathologists was only fair to good with a kappa score of 0.56-0.61. ${ }^{25}$ It can be anticipated that interobserver agreement would be worse if such a study were done with biopsy specimens only.

Uncertainty also exists regarding the natural history of high grade dysplasia. Recent studies described a cancer incidence of $14-56 \%$ at three years and 59\% at five years. ${ }^{12}{ }^{13} 15$ This concurs with previous estimates that suggest a high risk of progression of high grade dysplasia to adenocarcinoma. However, Schnell et al reported that only $16 \%$ of their patients with high grade dysplasia developed cancer at a mean follow up of 7.3 years. ${ }^{14}$ Others have reported regression of high grade dysplasia over time. ${ }^{12}{ }^{14}$ Thus high grade dysplasia remains a worrisome lesion, although progression to carcinoma may take many years and is not inevitable.
It is unclear why a subset of Barrett's patients with high grade dysplasia go on to develop cancer and how to best identify them. To date, only flow cytometry and $17 \mathrm{p}$ (p53) loss of heterozygosity have been identified as predictors of progression to adenocarcinoma in these patients. ${ }^{15}{ }^{26}$ The mortality of oesophagectomy is approximately $2 \%$ in expert hands and morbidity associated with the operation may impair quality of life. $^{1927}$ Thus better clinical and biological markers as predictors of cancer risk in patients with high grade dysplasia are needed, given the morbidity and mortality associated with oesophagectomy.

Limited information exists on the relationship between extent of high grade dysplasia and prevalence of unsuspected cancer at oesophagectomy. Intuitively, one would expect a greater extent of high grade dysplasia to be associated with a greater risk of cancer. Only two other studies have examined the relationship between extent of high grade dysplasia and adenocarcinoma risk. Weston et al described 15 patients with focal high grade dysplasia, defined as a single Barrett's oesophagus mucosal segment or single biopsy specimen with high grade dysplasia, of whom four $(26.7 \%)$ progressed to cancer over a period of three years. ${ }^{12}$ A recent study from the Mayo Clinic suggested that the extent of high grade dysplasia correlates with cancer risk. ${ }^{13}$ Only $14 \%$ of patients with focal high grade dysplasia, defined as the presence of a high grade dysplasia limited to five or fewer crypts and associated tubuloalveolar acini in one biopsy specimen, developed adenocarcinoma at three years compared with 56\% with diffuse high grade dysplasia, defined as high grade dysplasia involving more than five crypts and associated tubuloalveolar acini in one endoscopic biopsy specimen or involvement of more than one biopsy specimen with any amount of high grade dysplasia. Neither of these studies followed all patients to oesophagectomy. In contrast, our study, based on oesophagectomy results, found that $57 \%$ of patients with high grade dysplasia had unsuspected cancer at the time of oesophagectomy. However, the extent of high grade dysplasia was not a reliable predictor of unsuspected adenocarcinoma at the time of oesophagectomy regardless of whether the Cleveland Clinic or Mayo Clinic criteria for extent of high grade dysplasia were used.

At the present time, there is no uniformly accepted definition of focal or diffuse high grade dysplasia. While both the Mayo Clinic and Cleveland Clinic definitions are arbitrary, the literature describing the pathology of high grade dysplasia does not emphasise the number of crypts involved by the dysplastic process. In fact, surface epithelial involvement by the dysplastic process is invariably emphasised, not involvement of crypts. ${ }^{24} 28$ The extent of dysplasia and carcinoma in resection specimens can be quite small. A mapping study of 19 patients who underwent surgery for high grade dysplasia found that the median surface area of intestinal metaplasia without dysplasia was $32 \mathrm{~cm}^{2}$ but only $1.1 \mathrm{~cm}^{2}$ for the seven cancers. ${ }^{8}$ Further evidence for the distribution of cancer in patients with high grade dysplasia comes from Reid et al who found that cancer detected during endoscopic surveillance of high grade dysplasia was localised to a single $1 \mathrm{~cm}$ interval of the oesophagus in $82 \%$ of patients. ${ }^{23}$

Our study has several limitations. Firstly, our results may be biased by a longstanding institutional preference for surgery in patients with Barrett's oesophagus and high grade dysplasia. Secondly, the small sample size of this study may have caused a type II error, especially when using the Mayo Clinic criteria. Using the Cleveland Clinic criteria, patients with diffuse high grade dysplasia were more likely to have unsuspected cancer than individuals with focal high grade dysplasia. This trend may be statistically significant with a larger sample size. However, the clinical importance of such a finding would be limited as nearly $50 \%$ of our patients with focal high grade dysplasia had adenocarcinoma at the time of oesophagectomy. Nevertheless, this is one of the largest studies of oesophagectomy for high grade dysplasia to date. 
Finally, the biopsy protocol for patients with high grade dysplasia changed over time. The use of regular biopsy forceps was replaced with the large capacity forceps in 1993. Only since 2000 did we adopt the aggressive biopsy protocol advocated by Reid et al, consisting of four quadrant biopsies at $1 \mathrm{~cm}$ intervals in conjunction with biopsy of any mucosal abnormality. ${ }^{23}$ Thus it is conceivable that more occult cancers may have been detected if the biopsy protocol advocated by Reid et al had been used in all of these patients.

In summary, both focal and diffuse high grade dysplasia are risk factors for unsuspected cancer at the time of oesophagectomy, regardless of the definition used. This study suggests that there is no evidence, as yet, that the extent of high grade dysplasia can be used as a basis for decision making in Barrett's oesophagus patients with high grade dysplasia.

\section{ACKNOWLEDGEMENTS}

We wish to thank Tina Ours, RN, for statistical assistance and Michael Vaezi, MD, for reviewing this manuscript.

\section{Authors' affiliations}

M S Dar, G W Falk, Department of Gastroenterology and Hepatology, Center for Swallowing and Esophageal Disorders, Cleveland Clinic Foundation, Cleveland, Ohio, USA

J R Goldblum, Department of Anatomic Pathology, Center for Swallowing and Esophageal Disorders, Cleveland Clinic Foundation, Cleveland, Ohio, USA

T W Rice, Department of Thoracic and Cardiovascular Surgery, Center for Swallowing and Esophageal Disorders, Cleveland Clinic Foundation, Cleveland, Ohio, USA

\section{REFERENCES}

1 Lee RG. Dysplasia in Barrett's esophagus. A clinicopathologic study of six patients. Am J Surg Pathol 1985:9:845-52.

2 Altorki NK, Sunagawa M, Little AG, et al. High-grade dysplasia in the columnar- lined esophagus. Am J Surg 1991;161:97-100.

3 Miros M, Kerlin P, Walker N. Only patients with dysplasia progress to adenocarcinoma in Barrett's esophagus. Gut 1991;32:1441-6.

4 Pera M, Trastek VF, Carpenter HA, et al. Barrett's esophagus with high-grade dysplasia: an indication for esophagectomy? Ann Thorac Surg 1992;54:199-204.

5 Rice TW, Falk GW, Achkar E, et al. Surgical management of high-grade dysplasia in Barrett's esophagus. Am J Gastroenterol 1993;88:183236

6 Edwards MJ, Gable DR, Lentsch AB, et al. The rationale for esophagectomy as the optimal therapy for Barrett's esophagus with high-grade dysplasia. Ann Surg 1996:223:585-91.

7 Heitmiller RF, Redmond M, Hamilton SR. Barrett's esophagus with high-grade dysplasia: an indication for prophylactic esophagectomy. Ann Surgery 1996;224:66-71.
8 Cameron AJ, Carpenter HA. Barrett's esophagus, high-grade dysplasia, and early adenocarcinoma: a pathologic study. Am J Gastroenterol 1997:92:586-91.

9 Ferguson MK, Naunheim KS. Resection for Barrett's mucosa with high-grade dysplasia: implications for prophylactic photodynamic therapy. J Thorac Cardiovasc Surg 1997; 114:824-49.

10 Falk GW, Rice TW, Goldblum JR, et al. Jumbo biopsy forceps protocol still misses unsuspected cancer in Barrett's esophagus with high-grade dysplasia. Gastrointestinal Endosc 1999:49:170-6.

11 Zaninotto G, Parenti AR, Ruol A, et al. Oesophageal resection for high-grade dysplasia in Barrett's oesophagus. Br J Surg 200;87: 1102-5

12 Weston AP, Sharma P, Topalovski M, et al. Long-term follow-up of Barrett's high- grade dysplasia. Am J Gastroenterol 2000;95:1888-93.

13 Buttar NS, Wang KK, Sebo TJ, et al. Extent of high-grade dysplasia in Barrett's esophagus correlates with risk of adenocarcinoma. Gastroenterology 2001;120:1630-9.

14 Schnell TG, Sontag SJ, Chejfec G, et al. Long-term nonsurgical management of Barrett's esophagus with high-grade dysplasia. Gastroenterology 2001;120:1607-19

15 Reid BJ, Levine DS, Longton G, et al. Predictors of progression to cancer in Barrett's esophagus: baseline histology and flow cytometry identify low- and high-risk patient subsets. Am J Gastroenterol 2000;95: 1669-76.

16 Levine DS, Haggitt RC, Blount PL, et al. An endoscopic biopsy protocol can differentiate high-grade dysplasia from early adenocarcinoma in Barrett's esophagus. Gastroenterology 1993;105:40-50.

17 Overholt BF, Panjehpour M, Haydek JM. Photodynamic therapy for Barrett's esophagus: follow-up in 100 patients. Gastrointest Endosc 1999:49:1-7.

18 Gossner L, Stolte M, Sroka R, et al. Photodynamic ablation of high-grade dysplasia and early cancer in Barrett's esophagus by means of 5-aminolevulinic acid. Gastroenterology 1998;114:448-55.

19 Rice TW, Blackstone EH, Goldblum JR, et al. Superficial adenocarcinoma of the esophagus. J Thorac Cardiovasc Surg 2001;122:1077-90.

20 Holscher AH, Bollschweiler, Schneider PM, et al. Prognosis of early esophageal cancer. Comparison between adeno- and squamous cell carcinoma. Cancer 1995;76:178-86.

21 Sabik JF, Rice TW, Goldblum JR, et al. Superficial esophageal carcinoma. Ann Thorac Surg 1995;60:896-902.

22 Pellegrini CA, Pohl D. High-grade dysplasia in Barrett's esophagus: surveillance or operation? J Gastrointest Surg 2000:4:131-4.

23 Reid BJ, Blount PL, Feng Z, et al. Optimizing endoscopic biopsy detection of early cancer in Barrett's high-grade dysplasia. Am Gastroenterol 2000;95:3089-96

24 Montgomery E, Bronner MP, Goldblum JR, et al. Reproducibility of the diagnosis of dysplasia in Barrett's esophagus: a reaffirmation. Hum Pathol 2001:32:368-78.

25 Ormsby $\mathbf{A H}$ Petras RE, Hendricks $\mathrm{WH}$ et al Interobserver variation in Barrett's related high grade dysplasia and superficial carcinoma: can it be improved using uniform pathological criteria? Gut 2002;51:671-6.

26 Reid BJ, Prevo L, Galipeau PC, et al. Predictors of progression in Barrett's esophagus II: baseline 17p (p53) loss of heterozygosity identifies a patient subset at increased risk for neoplastic progression. Am J Gastroenterol 2001;96:2839-48.

27 Kirby JD. Quality of life after esophagectomy: the patients' perspective. Dis Esophagus 1999;12:168-71

28 Haggitt RC. Barrett's esophagus, dysplasia, and adenocarcinoma. Hum Pathol 1994;25:982-93 